Corrigendum

\title{
Corrigendum to "Nanoporous Ag-Au Bimetallic Triangular Nanoprisms Synthesized by Galvanic Replacement for Plasmonic Applications"
}

\author{
Hongmei Qian (D), ${ }^{1}$ Shoaib Anwer, ${ }^{2}$ G. Bharath, ${ }^{3}$ Shahid Iqbal, ${ }^{4}$ and Lijuan Chen ${ }^{5}$ \\ ${ }^{1}$ Department of Architecture and Civil Engineering, West Anhui University, Anhui 237012, China \\ ${ }^{2}$ Department of Mechanical Engineering, Khalifa University of Science and Technology, Abu Dhabi 127788, UAE \\ ${ }^{3}$ Department of Chemical Engineering, Khalifa University of Science and Technology, Abu Dhabi 127788, UAE \\ ${ }^{4}$ School of Chemistry and Chemical Engineering, University of Chinese Academy of Sciences, Beijing 100049, China \\ ${ }^{5}$ College of Materials and Chemical Engineering, West Anhui University, Anhui 237012, China
}

Correspondence should be addressed to Hongmei Qian; hmqian0621@163.com

Received 1 October 2018; Accepted 18 October 2018; Published 29 October 2018

Copyright (C) 2018 Hongmei Qian et al. This is an open access article distributed under the Creative Commons Attribution License, which permits unrestricted use, distribution, and reproduction in any medium, provided the original work is properly cited.

In the article titled "Nanoporous Ag-Au Bimetallic Triangular Nanoprisms Synthesized by Galvanic Replacement for Plasmonic Applications" [1], the affiliation of the fourth author was incorrect. The corrected authors' list and affiliations are shown above.

\section{References}

[1] H. Qian, S. Anwer, G. Bharath, S. Iqbal, and L. Chen, "Nanoporous Ag-Au bimetallic triangular nanoprisms synthesized by galvanic replacement for plasmonic applications," Journal of Nanomaterials, vol. 2018, Article ID 1263942, 7 pages, 2018. 


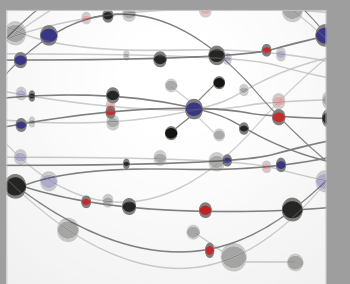

The Scientific World Journal
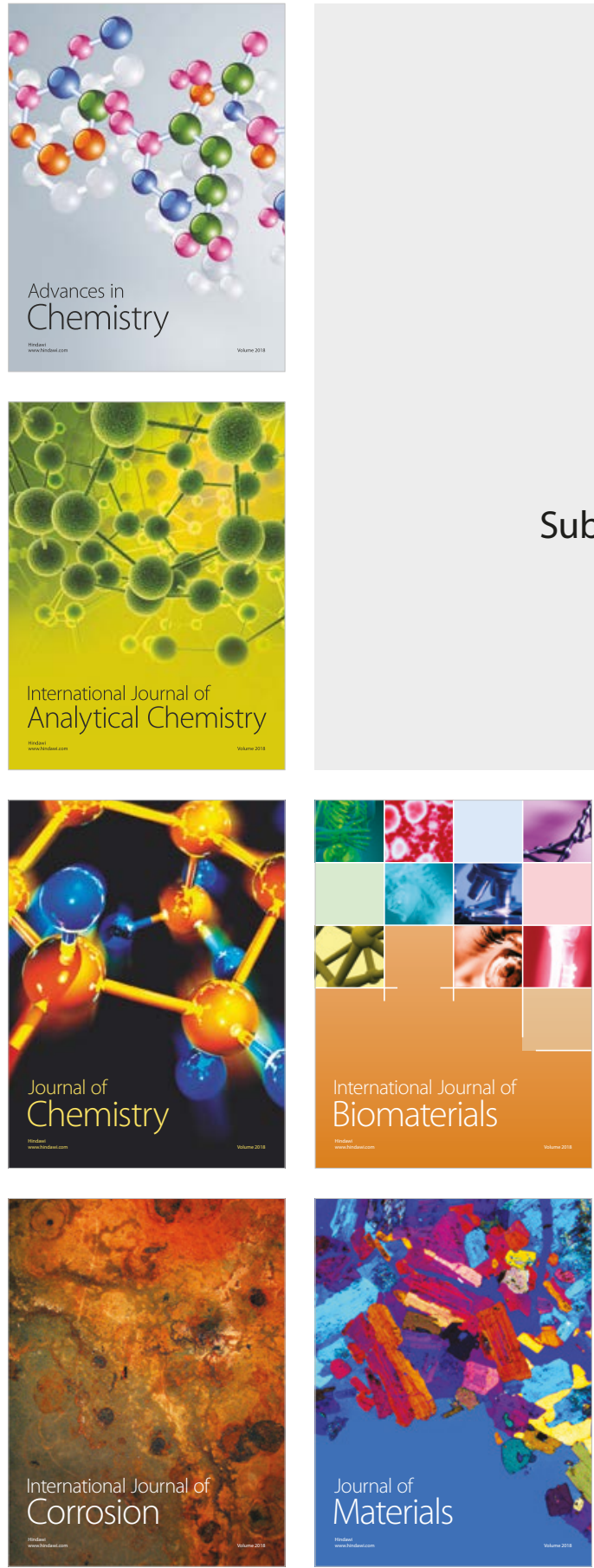

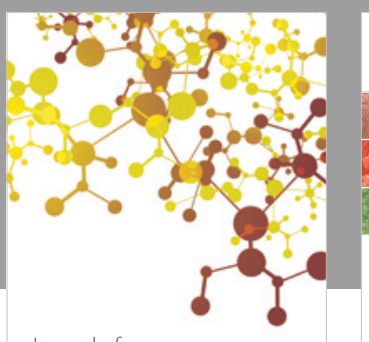

Journal of

Applied Chemistry
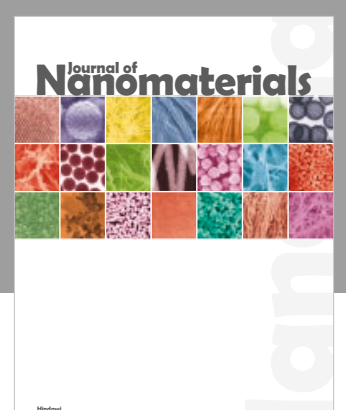

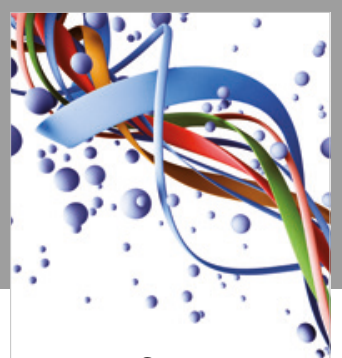

Scientifica

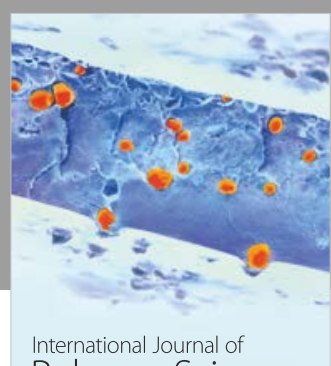

Polymer Science

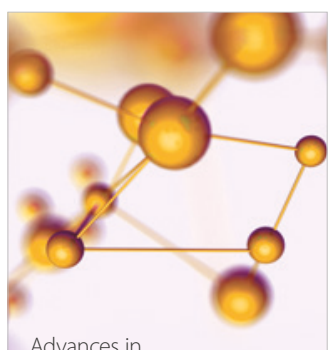

Physical Chemistry
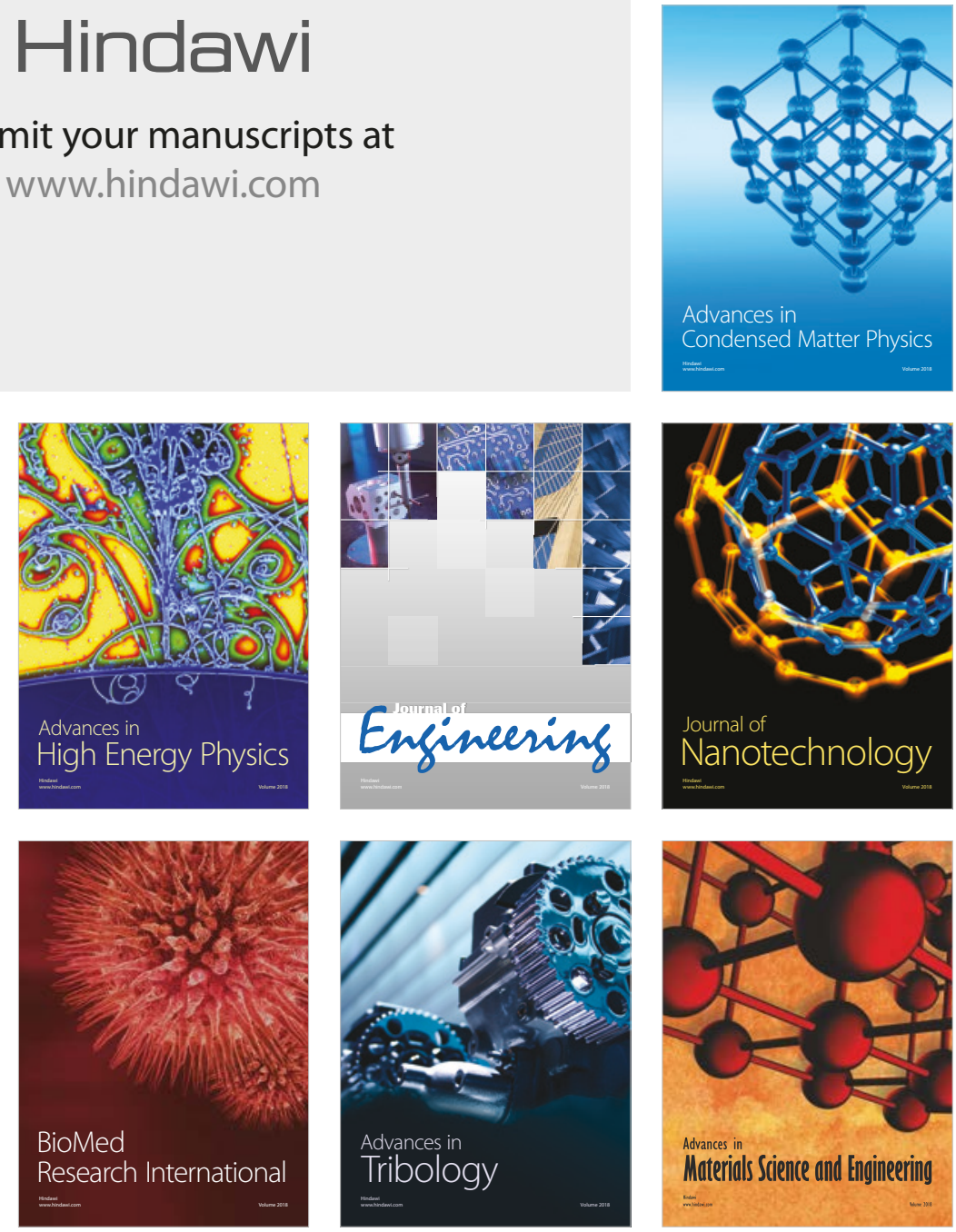